7. Stead RJ, Cooke NJ. Adverse effects of inhaled corticosteroids. BMJ 1989;298:403-4.

R.M. Stanbury

A.H. Chignell

E.M. Graham

Ophthalmology Department

St Thomas' Hospital

Lambeth Palace Road

London SE1 7EH, UK

Tel: $+44(0) 1719289292$, ext. 3135

Fax: $+44(0) 1719228165$

Sir,

\section{Vascular loop the loop}

We report the case of a 34-year-old man in whom a thick epiretinal membrane was found in association with a branch retinal vein occlusion, distal to an arteriovenous crossing abnormality.

\section{Case report}

A 34-year-old man was referred to the vitreo-retinal clinic with a 4 year history of blurred vision and macropsia in the left eye. He was previously fit and well, with no relevant ophthalmic history.

On examination, the visual acuity in the right eye was $6 / 4$ unaided, and in the left was 6/9. Both anterior segments were normal, as was the right fundus. However, the left fundus revealed a thick epiretinal membrane over the superotemporal arcade. Proximal to this, an arteriovenous crossing abnormality was noted; the vein completely looped around the artery (Fig. 1, arrow). A fluorescein angiogram confirmed the hypothesis of a branch retinal vein occlusion distal to the arteriovenous crossing abnormality. Fig. 1 shows the fluorescein angiogram taken in the early venous phase. Capillary drop-out and dilatation are revealed around the fovea, with straightening of the retinal blood vessels in this vicinity. Tortuosity of the superotemporal branch artery is apparent under the epiretinal membrane, which occupies approximately four disc areas above the fovea. There is collateral circulation involving branches of the

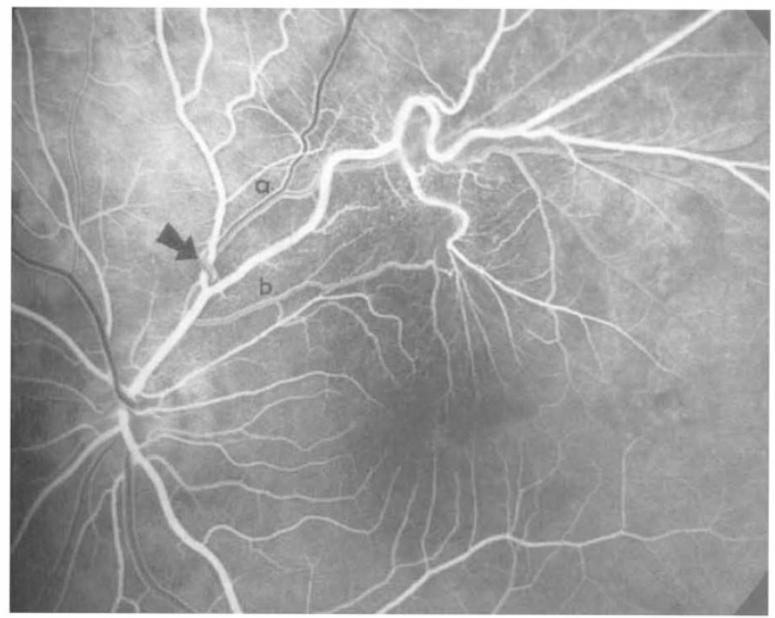

rig. 1. Fluorescein angiogram taken in the early venous phase. For details see text. superotemporal branch artery and vein, such that the veins accompanying the branch artery fill before the rest of the venous system (Fig. 1, labels ' $a$ ' and ' $b$ ').

The clinical observation of the epiretinal membrane and the findings of the fluorescein angiogram are consistent with a diagnosis of branch retinal vein occlusion. The patient was normotensive and no systemic predisposition to venous occlusive disease was identified.

\section{Comment}

Branch retinal vein occlusion is one of a number of causes of epiretinal membrane formation. ${ }^{1}$ Others include surgical procedures such as cataract extraction, scleral buckling, retinal argon laser and cryopexy, together with chronic intraocular inflammation, diabetic retinopathy and blunt trauma. Approximately $60 \%$ of vein occlusions occur in the superotemporal quadrant, ${ }^{2}$ with another $39 \%$ in the inferotemporal quadrant. They usually occur at arteriovenous crossings, and as there are more superotemporal crossings above the disc, the risk may be greater here. In addition, a greater proportion of these crossings have the artery passing over the vein, socalled artery-over-vein type. ${ }^{3}$ Zhao et al. ${ }^{+}$Found that $99 \%$ of vein occlusions occurred at artery-over-vein crossings, suggesting that a mechanical obstruction of the vein may have a role to play in the formation of thrombus. Seitz ${ }^{5}$ postulated that changes in the distension of the vein passing under the artery as opposed to over it resulted in haemodynamic differences between the two types of crossing. Turbulence secondary to reduced lumen diameter can cause intimal damage and initiate clotting. Klein" concluded that an increase in arteriovenous crossings, or congenital loop the loops in an otherwise normal fundus, could increase the risk of branch retinal vein occlusion. As yet, however, a complete loop the loop of vein and artery has not been described.

\section{References}

1. Appiah AP. Secondary causes of preretinal fibrosis. Ophthalmology 1975;96:389-97.

2. Weinberg D, Dodwell DG, Fern SA. Anatomy of arteriovenous crossings in branch retinal vein occlusion. Am J Ophthalmol 1990;109:298-302.

3. Wienberg D, Egan KM, Seddon JM. Asymmetrical dístribution of arteriovenous crossings in the normal retina. Ophthalmology 1993;100:31-6.

4. Zhao J, Sastry SM, et al. Arteriovenous crossing patterns in branch retinal vein occlusion. Ophthalmology 1993; 100:423-38.

5. Seitz R. The crossing phenomenon in the retina. St Louis: CV Mosby, 1964:20-74

6. Klein I. Prevention of retinal vein occlusion. Am J Ophthalmol 1950;33:175-89.

Anne Cook

D. Wong

B. MCNeela

St Paul's Eye Department

8 Link

Royal Liverpool University Hospital

Prescot Street

Liver pool L7 8XP

UK 